Revista de Investigación Educativa 22

enero-junio, 2016 | ISSN 1870-5308 | Xalapa, Veracruz

Instituto de Investigaciones en Educación | Universidad Veracruzana

\title{
Educación Superior y pueblos indígenas en América Latina. Contexto y experiencias
}

\author{
Mtra. Inés Olivera Rodríguez \\ Doctorante \\ Facultad de Filosofía y Letras, Universidad Nacional Autónoma de México \\ inesolivera@gmail.com
}

Mato, D. (Coord.). (2015). Educación Superior y pueblos indígenas en América Latina. Contexto y experiencias. Argentina: Universidad Nacional de Tres de Febrero. ISBN 978-987-1889-57-o. pp. 248.

Educación superior y pueblos indígenas en América Latina. Contexto y experiencias es un libro editado por la Universidad Nacional de Tres de Febrero (UnTREF) en Argentina. El libro, coordinado por Daniel Mato, se compone de diez artículos que aportan al conocimiento de la situación de los pueblos indígenas en la educación superior en América Latina. Organizado en tres partes, este volumen comienza con un análisis del panorama regional latinoamericano, acceso y permanencia de población indígena a/en educación superior, seguido por una revisión de los contextos nacionales de Bolivia, Ecuador, Brasil, Argentina y Chile, para finalizar con el análisis de experiencias específicas en Argentina, Brasil, Colombia, Perú y México.

El programa Educación Superior y Pueblos Indígenas y Afrodescendientes en América Latina (ESIAL) del Centro Interdisciplinario de Estudios Avanzados (CIEA) de la UNTREF fue creado en el año 2012 retomando la experiencia desarrollada, desde julio de 2007, por el proyecto Diversidad Cultural e Interculturalidad en Educación Superior en América Latina del IESALC-UnesCo; ambos programas bajo la coordinación de Daniel Mato. El libro que aquí se reseña representa la continuidad en el análisis y presentación de más de 200 experiencias latinoamericanas de acceso, per- 
manencia y graduación de estudiantes indígenas y afrodescendientes identificadas desde 2007; algunas de las cuales fueron presentadas y discutidas en los cinco libros publicados por IESALC-UNESCO entre 2008 y 2012.

Los trabajos aquí comprendidos fueron presentados y discutidos, en una primera versión, en el Coloquio y Taller: "Educación Superior y Pueblos Indígenas en América Latina. Contextos, Experiencias y Desafíos", realizado el 28 y 29 de abril del 2014 en la Ciudad de Buenos Aires. Dicho evento fue organizado por diversas instancias de la Universidad Nacional de Tres de Febrero (UNTREF) y contó con el auspicio del Instituto Internacional de la UNESCO para la Educación Superior en América Latina y el Caribe (UneCo-IESALC). En este evento se contó con la presentación de cinco investigadores/as procedentes de pueblos indígenas de Argentina, Brasil, Chile, Bolivia y Ecuador, y de cuatro investigadores posdoctorales de la UNT REF de Brasil, Argentina, Colombia y México. Estos aportes, además del artículo de visión regional realizado por Daniel Mato, componen el presente volumen.

Todos los textos de este volumen recuperan la legislación internacional sobre el tema. Especialmente los trabajos de Mato (pp. 19-44) y Mateos Cortés (pp. 181-206), traen un listado detallado del marco jurídico internacional. Desde la Convención Internacional para la Eliminación de todas las Formas de Discriminación Racial de 1965, pasando por el Convenio 169 de la OIT firmado por primera vez en 1989, hasta la Declaración Universal sobre los Derechos de los Pueblos Indígenas de 2007, los/ as autores/as mencionados identifican más de diez documentos internacionales que amparan la necesidad y legalidad de promover una educación superior intercultural que garantice acceso de población indígena y afrodescendiente y que sea capaz de plantear modelos educativos que reconozcan la validez de sus saberes y formas de conocimiento.

Los marcos legales nacionales reconocen también la necesidad e importancia de la educación intercultural a todos los niveles; específicamente en las constituciones de los ocho países analizados en el presente volumen, que desde las reformas llevadas a cabo en la década de 1990 (1988 en el caso brasilero), se reconoce su condición intercultural o plurinacional. A este respecto los trabajos de Choque Quispe sobre Bolivia (pp. 47-72), Cuji sobre Ecuador (pp. 73-96), Millán sobre Argentina (pp. 117-134), Gomes do Nascimento sobre Brasil (pp. 97-116), Mora Curriao sobre Chile (pp. 135-156) y de Santamaría Chavarro sobre Colombia (pp. 223-244) presentan los marcos legales nacionales, analizando además los avances y retrocesos de dicho desarrollo jurídico. 
Sin embargo, como señala Daniel Mato en el primer artículo de este volumen, a pesar de los avances constitucionales y legislativos que, debe ser dicho, han sido impulsados por las luchas de los pueblos indígenas y afrodescendientes, los cambios en los sistemas educativos son aún insuficientes para garantizar la equidad. El autor marca un contrapunto importante: si bien los avances se ven limitados y llenos de contradicciones las modificaciones en los marcos constitucionales representan un momento de cambio que abre diversas posibilidades. En casos como el mexicano, el brasilero y el chileno, analizados en este volumen, los cambios constitucionales impulsaron la sanción de legislación que posibilitó la creación de programas de inclusión por becas y cuotas de ingreso, además de la creación de instituciones de educación superior interculturales.

Además de los aportes a partir de la presentación de los marcos legales, se pasa a señalar otros dos aspectos de discusión de central importancia para el debate actual: En primer lugar, el punto de análisis común a este conjunto de investigadores/as es el tema de la equidad desde el reconocimiento de la herencia colonial que caracteriza a la región. La herencia colonial, conceptualizada en América Latina como colonialidad del poder, ha sido el elemento que históricamente ha mantenido al margen de derechos y ciudadanía a los pueblos indígenas y afrodescendientes, no solo en términos demográficos, sino también epistemológicos. En este sentido, como señalan los autores del presente volumen, la demanda por interculturalidad en la educación superior, no supone apenas el acceso de población indígena y afrodescendiente a las universidades sino que implica propuestas educativas que sean capaces de partir de un diálogo de saberes (Mateos Cortés) para construir conocimiento a partir de la colaboración intercultural (Santos Fernandes). La apuesta es por una interculturalización de la educación superior, más allá de la inclusión de población excluida (Mato).

La crítica a la herencia colonial y a sus impactos en la educación formal pasa por el reconocimiento de la necesidad de buscar modelos de vida alternativos al desarrollo; sobre esto, algunos trabajos se centran en las posibilidades de categorías como Buen Vivir. En el caso boliviano Choque Quispe recoge de forma recurrente los elementos centrales que ha tenido la educación intercultural en Bolivia donde se defiende que la educación debe ser "comunitaria, intercultural y productiva", además de descolonizadora, lo que debe basarse en una reconstrucción de las formas de vida comunitarias.

Los trabajos de Choque Quispe y de Cuji, evidencian las contradicciones que se generan al intentar formalizar los programas interculturales, siendo la princi- 
pal de ellas la discusión en torno a la calidad. Así, el segundo aspecto transversal a los textos es el de calidad. Según Choque Quispe, Cuji y Mato: si bien en algunos casos se logra construir y formalizar una propuesta de educación superior intercultural, se logra el reconocimiento de sus planes de trabajo, de sus carreras interculturales, de sus formas de enseñanza en alternancia, semi-presenciales, en base a saberes indígenas, con presencia de maestros y sabios de la comunidad, en la evaluación de la calidad de estas propuestas no se contempla la particularidad de sus proyectos. Analizando el caso de la desaparecida universidad Amawtay Wasi en el Ecuador, Luis Fernando Cuji señala el desencuentro entre las propuestas pedagógicas interculturales elaboradas desde lo simbólico y las lógicas de evaluación pensadas desde lo medible.

El avance en la consolidación de algunas propuestas de educación superior e interculturalidad hace urgente pensar en otro momento de la educación intercultural: el de su institucionalización y formalización, pasando por los sistemas de evaluación que le permiten acceder a más y mejores recursos y reconocimientos. En este sentido, lo urgente y pendiente de la agenda de la educación superior intercultural es la evaluación pertinente. Como señala Mato:

La evaluación de los programas, universidades y otros tipos de IEs interculturales y/o indígenas, como la de sus docentes e investigadores, requiere diseñar instrumentos que tomen en cuenta la especificidad de las respectivas propuestas institucionales, así como la de los contextos en que se desarrollan. Es necesario revisar los criterios de evaluación y acreditación para asegurar que estos respondan a las peculiaridades de los diversos tipos de Universidades y otros tipos de IES, con especial atención a diferencias asociadas a particularidades lingüísticas, socioculturales, territoriales y otras significativas, según la diversidad cultural propia de cada país. (p. 40)

Finalmente, el artículo de Mateos Cortés sobre la Universidad Veracruzana Intercultural presenta un interesante foco de análisis que se quiere aquí subrayar. En este trabajo el abordaje desde los/as egresadas permite enfocar lo que tiene de diferenciadora la educación superior intercultural. La lucha por la implementación y ampliación de oferta de educación intercultural, y la necesidad de debate y reflexión en torno a la configuración de programas educativos "verdaderamente" interculturales, han tomado, por necesidad, la mayor parte del debate sobre el tema. En este contexto, ha sido difícil identificar y resaltar en los resultados (egresados y egresadas) lo diferencial de la propuesta intercultural en la educación superior. Como se señala en este volumen, 
si la educación intercultural no es solo acceso sino proyecto, este proyecto debe ser disidente frente a los programas.

En este trabajo de reseña la atención se ha concentrado en algunos temas transversales que evidencian los nudos y puntos álgidos del debate actual sobre educación superior e interculturalidad. A este respecto, los diez artículos contenidos en este volumen representan importantes aportes al conocimiento y debate sobre el tema. Estos aportes se pueden identificar a varios niveles: desde el manejo de la normatividad global y local, pasando por el análisis teórico que recoge conceptos densos y críticos desde la constatación de la colonialidad del poder, recogiendo el análisis histórico y político que permite una mirada crítica sobre los procesos de construcción de la educación y los pueblos indígenas en la región, para finalmente aportar con reflexiones en torno a los pendientes en las instituciones interculturales y en el debate teórico que las acompaña. En este sentido, el presente volumen es un aporte fundamental al debate y estado de la cuestión en el tema. 